\title{
Distinct functions of homeodomain- containing and homeodomain-less isoforms encoded by homothorax
}

\author{
Barbara Noro, ${ }^{1}$ Joaquim Culi, ${ }^{3}$ Daniel J. McKay, ${ }^{2}$ Wen Zhang, ${ }^{1}$ and Richard S. Mann ${ }^{1,4}$ \\ ${ }^{1}$ Department of Biochemistry and Molecular Biophysics, ${ }^{2}$ Integrated Program in Cellular, Molecular, and Biophysical \\ Studies, Columbia University, New York, New York 10032, USA; ${ }^{3}$ Centro de Biología Molecular Severo Ochoa, Consejo \\ Superior de Investigaciones Científicas (CSIC) and Universidad Autónoma de Madrid, 28049 Madrid, Spain
}

The homothorax (hth) gene of Drosophila melanogaster is required for executing Hox functions, for head development, and for forming the proximodistal (PD) axis of the appendages. We show that alternative splicing of $h$ th generates two types of protein isoforms, one that contains a DNA-binding homeodomain (HthFL) and one that does not contain a homeodomain (HDless). Both types of Hth isoforms include the evolutionarily conserved HM domain, which mediates a direct interaction with Extradenticle (Exd), another homeodomain protein. We show that although both HthFL and HDless isoforms of Hth can induce the nuclear localization of Exd, they carry out distinct sets of functions during development. Surprisingly, we find that many of hth's functions, including PD patterning and most Hox-related activities, can be executed by the HDless isoforms. In contrast, antennal development shows an absolute dependency on the HthFL isoform. Thus, alternative splicing of $h t h$ results in the generation of multiple transcription factors that execute unique functions in vivo. We further demonstrate that the mouse ortholog of hth, Meis1, also encodes a HDless isoform, suggesting that homeodomain-less variants of this gene family are evolutionarily ancient.

[Keywords: homothorax; homeodomain; Hox; selector gene; Drosophila melanogaster]

Supplemental material is available at http://www.genesdev.org.

Received January 24, 2006; revised version accepted April 11, 2006.

Multicellular organisms show an astounding degree of morphological and behavioral complexity, a reflection of their proteome size and the intricacy of interactions between proteome components. The total number of genes in an organism represents only the baseline upon which different mechanisms act to increase coding capacity. Indeed, the number of distinct functional proteins can exceed the number of genes by orders of magnitude, due to biological processes such as DNA recombination, mRNA processing, and post-translational protein modification (Maniatis and Tasic 2002). Alternative premRNA splicing is thought to have one of the most widespread roles in generating protein diversity, in terms of both the number of affected genes and the range of organisms in which it occurs. The diversity that may be produced by alternative splicing ranges from subtle differences in protein activity to the generation of isoforms with antagonistic functions (Lopez 1995; Graveley 2001; Maniatis and Tasic 2002).

Drosophila melanogaster displays complex developmental, morphological, and behavioral traits, despite a

${ }^{4}$ Corresponding author.

E-MAIL rsm10@columbia.edu; FAX (212) 305-7924.

Article is online at http://www.genesdev.org/cgi/doi/10.1101/gad.1412606. genome of only $\sim 14,000$ genes (Adams et al. 2000; Celniker and Rubin 2003). Spectacular examples of diversity generated by alternative splicing have been observed in D. melanogaster, such as encoded by the gene Downs syndrome cell adhesion molecule (Dscam). This axon guidance receptor gene can potentially generate up to 38,016 isoforms, two to three times the number of annotated genes in the genome (Schmucker et al. 2000; Wojtowicz et al. 2004).

Alternative splicing also provides additional complexity to genes encoding nuclear transcription factors (Read and Manley 1992; Lopez 1995; Bayer et al. 1996; Goeke et al. 2003). Transcription factors are highly modular in nature, often containing separable domains for DNA binding, dimerization, subcellular localization, and transcriptional activation or repression. Alternative splicing operates on this modularity to expand genomic coding capacity, producing isoforms with potentially subtle or highly diverse functional differences. In principle, transcription factor variants could impact widely on the regulation of transcriptional networks, providing a means for fine-tuning the system as well as creating additional functional diversity. However, the relevance of the majority of alternative splicing events remains elusive, as distinct roles of alternatively spliced isoforms are 
typically not well established but only inferred from variations in predicted protein sequences.

Here we demonstrate that alternative splicing at the homothorax (hth) locus of Drosophila leads to the generation of multiple protein isoforms that carry out distinct functions in vivo. Previous analyses of $h t h$ demonstrated that it encodes a homeodomain (HD) transcription factor of the three-amino-acid extension loop (TALE) subfamily, homologous to the vertebrate Meis and Prep proteins (Burglin 1997; Rieckhof et al. 1997; Pai et al. 1998). This subfamily of homeodomain proteins displays a high degree of conservation, both within the $\mathrm{HD}$ and in an N-terminal Hth/Meis (HM) domain (Rieckhof et al. 1997; Pai et al. 1998). The HD is responsible for DNA binding while the HM domain mediates direct interactions with members of another TALEhomeodomain subfamily, Extradenticle (Exd) in Drosophila and Pbx in vertebrates (Knoepfler et al. 1997; Rieckhof et al. 1997; Berthelsen et al. 1998b; Ryoo et al. 1999).

In Drosophila, Exd is widely expressed throughout development, but its subcellular localization is tightly regulated by $\mathrm{Hth}$. In the absence of Hth, Exd resides in the cytoplasm, while in the presence of Hth, the proteins interact and translocate into the nucleus to regulate transcription. Importantly for the results described here, the HM domain of Hth is sufficient to translocate Exd from the cytoplasm to the nucleus (Abu-Shaar et al. 1999; Ryoo et al. 1999). The interaction between Exd and Hth has an additional consequence: Just as Exd's nuclear localization depends on Hth, Hth is unstable in the absence of Exd. These observations suggest that Hth and Exd are obligate partners that work together as a single functional unit. Consistent with this notion, identical phenotypes are generated by the lack of exd or hth function (Gonzalez-Crespo and Morata 1995; Rieckhof et al. 1997; Casares and Mann 1998, 2000; Abu-Shaar et al. 1999; Ryoo et al. 1999).

Hth and Exd are also intimately associated with the Hox proteins, another family of highly conserved homeodomain proteins that instruct many cell fate decisions during animal development (McGinnis and Krumlauf 1992). In the absence of Exd and/or Hth, Hox proteins are unable to regulate many of their target genes (Rauskolb and Wieschaus 1994; Ryoo and Mann 1999. Ryoo et al. 1999; Ebner et al. 2005). One reason for this link between Hth/Exd and Hox is that, for some target genes, Hth/Exd behaves as a Hox cofactor. Several examples of enhancers that bind Hox/Exd/Hth complexes have been described in which Hox DNA-binding selectivity and/or affinity are enhanced by Hth/Exd (Popperl et al. 1995; Maconochie et al. 1997; Jacobs et al. 1999; Ryoo et al. 1999; Gebelein et al. 2002, 2004; Ebner et al. 2005). Structural studies have shown that the Exd TALE peptide, a three-amino-acid insertion between helices 1 and 2 of its HD, mediates direct interactions with a conserved motif called YPWM that is present in most Hox proteins, enabling complex formation and cooperative DNA binding (Passner et al. 1999; Piper et al. 1999; LaRonde-LeBlanc and Wolberger 2003).
In addition to Hox-dependent activities, Hth and Exd have many Hox-independent functions during Drosophila development. For instance, they act as master regulators of the antennal developmental program, superimposing antennal identity on an appendage groundstate plan (Casares and Mann 1998, 2001; Dong et al. 2000, 2002). Hth and Exd are also required for leg and wing development, for the correct establishment of the proximal structures of these appendages (Abu-Shaar and Mann 1998; Wu and Cohen 1999, 2000; Azpiazu and Morata 2000, 2002; Casares and Mann 2000; Dong et al. 2001 ;).

In this study, we show that in addition to the previously characterized full-length protein (HthFL), alternative splicing of $h$ th generates two very similar isoforms that lack the HD but contain the HM domain (HDless). Hth HDless variants can interact with Exd and are extensively coexpressed with the HthFL isoform, implying that distinct Hth/Exd complexes are present in the same nuclei. Surprisingly, an analysis of hth mutant alleles that are unable to produce any Hth protein or can only express HDless isoforms demonstrate that the HD is dispensable for a substantial number of Hth functions during development, suggesting that the HDless variants are capable of correctly regulating many Hth target genes. The HD is essential, however, for a subset of Hth tasks, such as the instruction of antennal identity. These observations demonstrate that different Hth isoforms have unique transcriptional properties, which depend on the presence or absence of the HD. Thus, hth alternative splicing is used as a mechanism to expand the diversity of Hth/Exd complexes, increasing the complexity of the transcriptional networks that use these transcription factors. We also report that HDless isoforms are encoded by the vertebrate Meis1 gene, suggesting that this division of labor among HD-containing and HDless forms of this gene family may also exist in vertebrates.

\section{Results \\ homothorax encodes splice variants that lack a DNA-binding domain}

hth includes 16 annotated exons distributed over $>100$ kb of genomic DNA (Fig. 1A; FlyBase, http://www.flybase. org). All functionally characterized isoforms of $h t h$ include both the HM domain, encoded by exons 2-6, and the HD, encoded by exons 11-13 (Fig. 1A). In addition, $h t h$ encodes at least two additional alternatively spliced variants that have an intact HM domain but no HD. Both alternatively spliced mRNAs code for two almost identical HM-containing proteins that are largely derived from the first six coding exons. Both of these HDless isoforms have an additional 24 amino acids at their $\mathrm{C}$ termini encoded by alternate exons (Fig. 1A,C). One of these variants (the $7^{\prime}$ isoform) uses an alternative exon 7 (exon $7^{\prime}$ ). Sequence comparisons between $D$. melanogaster and Anopheles gambiae hth genes enabled us and others (Glazov et al. 2005) to identify a second hth splice variant that is also missing the $\mathrm{HD}$ (see also FlyBase, 

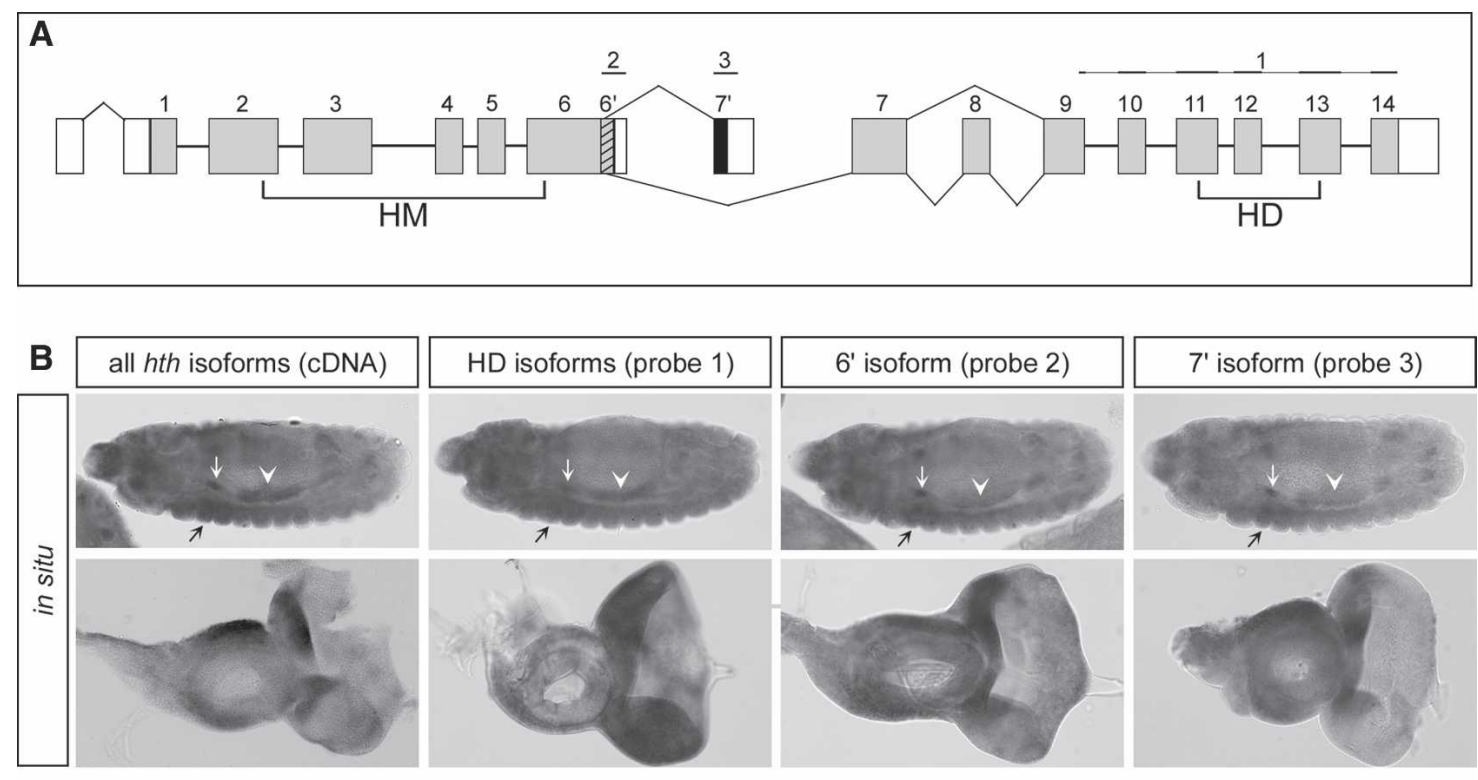

C

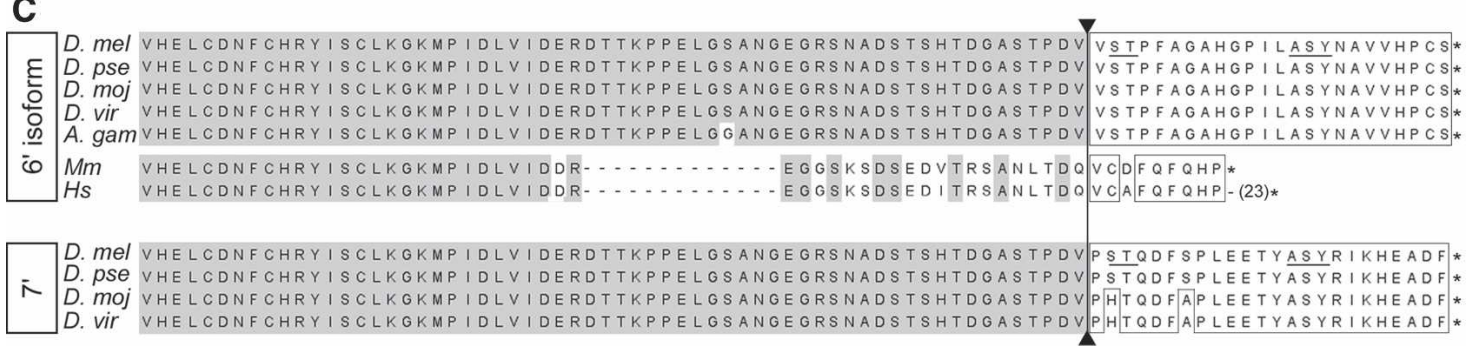

Figure 1. The $h t h$ locus encodes for evolutionary conserved HDless variants. (A) Genomic organization of hth (not shown to scale). White and gray boxes denote noncoding and protein-coding exons, respectively, while angled lines represent sites of alternative splicing. The $6^{\prime}$ - and $7^{\prime}$-specific ORFs are indicated by the dashed and black boxes, respectively. (B) In situ hybridization using probes for specific $h$ th isoforms shows very similar expression patterns during both embryonic (whole embryo, top panels) and larval (e.g., eye-antennal imaginal disc, bottom panels) stages. White and black arrows indicate high levels of hth expression in the gastric caeca and the thoracic ectoderm, respectively. White arrow heads point to hth staining in the visceral mesoderm. The positions of the probes used are indicated in $A$. See also Supplementary Figure 1. (C) Comparison of the predicted protein sequences of the $6^{\prime}$ and $7^{\prime}$ isoforms. The species indicated are $D$. melanogaster $(D$. mel), D. pseudoobscura (D. pse), D. mojavensis (D. moj), D. virilis (D. vir), A. gambiae (A. gam), M. musculus $(\mathrm{Mm})$, and Homo sapiens (Hs). The protein sequences of exon 6 and alternative exon 6 and 7 are compared. Amino acids conserved across all species are outlined by the gray shading. Sequence conservation for the $6^{\prime}$ and $7^{\prime}$ exons within the insect or mammal subgroups are boxed. The two black triangles demarcate the bypassed splice junction at the end of exon 6 . Underlined in black are two small motifs common to the $6^{\prime}$ and $7^{\prime}$ isoforms.

http://www.flybase.org). This isoform (the $6^{\prime}$ isoform) is generated when the splice site at the $3^{\prime}$ end of exon 6 is not used, generating an extended ORF (Fig. 1A,C). We confirmed the existence of both $6^{\prime}$ and $7^{\prime}$ isoforms in vivo by sequencing ESTs and performing RT-PCR on mRNA isolated from embryonic and larval tissues (data not shown). The presence of $6^{\prime}$ and $7^{\prime}$ isoforms raised the possibility that HDless variants of Hth might carry out distinct functions, suggesting a functional diversification of the $h$ th gene that depends on alternative splicing.

To test if the different isoforms have separate spatial or temporal expression profiles, we examined their distributions by in situ hybridization (Fig. 1B). All the probes revealed very similar expression patterns during both embryonic and larval stages, suggesting the coexpression of HthFL and HDless isoforms in the same tissues (Fig. 1B; data not shown). The in situ hybridization results are supported by immunohistochemical analysis of imaginal discs with antibodies that specifically recognize the HthFL and 6' isoforms (Supplementary Fig. 1).

\section{$6^{\prime}$ and $7^{\prime}$ HDless orthologs}

Comparative sequence analysis of the $h t h$ locus in other Drosophila species, including Drosphila pseudoobscura, Drosophila virilis, and Drosophila mojavensis, revealed a high degree of conservation of the alternative $6^{\prime}$ and $7^{\prime}$ exons, which translate into Hth proteins that are nearly identical to their D. melanogaster ortholog (Fig. 1C). The conservation of the 6' HDless form also extends to the mosquito A. gambiae, which diverged from Drosophila as long as 250 million years ago (Zdobnov et al. 2002). No analogous 7' exon could be identified in the Anopheles genome using BLAST searches. The high degree of con- 
servation observed among the Drosophila species, as well as in the mosquito (for the $6^{\prime}$ form), suggests that HDless Hth variants may exert conserved functions in Dipterans.

We further tested the evolutionary conservation of HDless isoforms by analyzing the genomic organization of Meis1, a vertebrate ortholog of $h t h$. Strikingly, the Meis1 intronic sequence immediately downstream of exon 6 is unusually conserved between the mouse and human genes. Moreover, if the splice site at the end of exon 6 is bypassed, as it is in Diptera, a HDless variant of Meis1 may be encoded (Fig. 1C). RT-PCR using mRNA from embryonic day 10.5 (E10.5) mouse embryos confirmed the presence of transcripts predicted to encode a HDless isoform that contains the first six exons and nine novel, additional amino acids (Fig. 1C). These additional residues are unrelated to the $24 \mathrm{C}$-terminal residues found in the Drosophila $6^{\prime}$ or $7^{\prime}$ isoforms. The presence of $6^{\prime}$ variants in both Diptera and vertebrates indicates a strong selective pressure to maintain both HD-containing and HDless isoforms of Hth/Meis, underscoring the functional significance of HDless variants in animal development.

The hth ${ }^{100-1}$ allele only expresses HDless isoforms that productively interact with Exd

To begin to address the role of HDless forms of Hth in vivo, we characterized two hth alleles in more detail. The first allele, $h t h^{\mathrm{P} 2}$, is a strong hypomorph, generated by a P-element insertion in the $h t h$ promoter. In homozygous $h t h^{\mathrm{P} 2}$ tissue, no Hth protein can be detected as assayed by immunostaining with an anti-Hth antibody that detects both HthFL and HDless isoforms (anti-Hth) (see below and Materials and Methods). The second allele, $h t h^{100-1}$, is predicted to encode only HDless isoforms due to an Arg321 to opal mutation in exon 9 (Kurant et al. 2001). We confirmed these predictions by carrying out immunoblot analyses with wild-type and mutant embryonic extracts. When probed with anti-Hth, wild-type extracts show two bands of the predicted sizes for the HthFL and HDless isoforms, respectively (Fig. 2A). Neither band is observed in $h t h^{\mathrm{P} 2}$ extracts (Fig. 2A). In $h t h^{100-1}$ extracts, no HthFL is observed but the faster migrating species is still present, accompanied by additional $h t h^{100-1}$-specific isoforms. These new variants are likely the result of the premature stop codon in exon 9 and are therefore predicted to be HM-containing, HDless forms with additional residues derived from the linker region between the HM and HD. These results suggest that hth expresses both HthFL and HDless isoforms. These two alleles provide valuable tools for comparing a strong loss-of-function state $\left(h t h^{\mathrm{P} 2}\right)$ versus a state in which only HDless forms are present $\left(h t h^{100-1}\right)$.

Because both HDless variants include an intact HM domain, which is necessary and sufficient to mediate an interaction with Exd (Ryoo et al. 1999), we predicted that they should both be able to interact with Exd. To test this prediction, we immunoprecipitated Exd from total embryonic extracts and probed these immunoprecipi- tates (IPs) with anti-Hth. Two bands having the predicted sizes for the HthFL and HDless isoforms are observed (Fig. 2B). To confirm that the slower, but not the faster, migrating band contains the Hth $\mathrm{HD}$, we also probed these IPs with an antibody that was generated against the $\mathrm{C}$-terminal HD-containing portion of $\mathrm{Hth}$ (anti-HthHD) (see Materials and Methods). Consistent with our predictions, this antibody detects the slower, but not the faster, migrating band (Fig. 2B).

We also confirmed that the HDless isoforms encoded by $h t h^{100-1}$ are able to translocate Exd into nuclei in vivo. As expected, no Hth or nuclear Exd can be detected in $h t h^{\mathrm{P} 2}$ mitotic clones (Fig. 2C). In contrast, $h t h^{100-1} \mathrm{mu}-$ tant clones present in proximal domains of the wing or leg imaginal discs have nuclear Exd (Fig. 2C; data not shown). Also as predicted, $h t h^{\mathrm{P} 2}$ clones show no immunostaining with the anti-HthHD antibody or with an antibody specifically raised against the unique sequences in exon 6' (anti-Hth6') (see Materials and Methods). In contrast, $h t h^{100-1}$ clones are stained with the antiHth6' antibody but not with the anti-HthHD antibody (Fig. 2D).

In summary, these data demonstrate that both fulllength and HDless isoforms of Hth can interact with and translocate Exd into nuclei. Further, consistent with the immunoblot analyses described above, these immunostaining experiments suggest that $h t h^{\mathrm{P} 2}$ makes no detectable Hth protein whereas $h t h^{100-1}$ produces only HDless isoforms.

\section{HDless forms of Hth are required for patterning the Drosophila embryo}

The $h t h^{100-1}$ allele allows us to examine a situation in which no full-length, HD-containing Hth isoforms are expressed. In contrast, there are no known $h t h$ alleles that express only HD-containing isoforms. Multiple attempts to use RNA interference (RNAi) hairpin constructs to specifically eliminate HDless isoforms in vivo failed, perhaps due to the very limited sequences available for targeting (data not shown). Instead, to mimic a HDless-depleted state in vivo, we codepleted the $6^{\prime}$ and $7^{\prime}$ isoforms by injecting small interfering RNAs (siRNAs) specifically directed against these splice variants into preblastoderm embryos. RT-PCR from a population of injected embryos revealed a significant reduction, but not complete loss, of both hth HDless transcripts (Fig. 3A; Supplementary Fig. 2A). No decrease was observed for the HthFL transcript, confirming the target specificity of the siRNAs (Fig. 3A). Consistently, siRNA-injected embryos show a selective loss of the HDless isoforms as detected by immunoblot analysis (Supplementary Fig. 2A).

Using these tools, we compared the contributions of HthFL and HDless isoforms to embryonic development. Toward the end of embryogenesis, the embryo secretes a cuticle, whose stereotyped features are a direct readout of patterning events that occurred during embryogenesis. $h t h$ is required both for proper segmentation and, as a Hox cofactor, to instruct segmental identities along the 
Figure 2. Hth HDless isoforms are capable of interacting with Exd. (A) Immunoblot of wild-type (wt), $h t h^{\mathrm{P} 2}$, and $h t h^{100-1}$ embryos stained with an anti-Hth antibody that detects all HM-containing isoforms $(\alpha-\mathrm{Hth})$. No Hth isoforms are detected in $h t h^{\mathrm{P} 2}$ embryos, while only putative HDless isoforms are present in $h t h^{100-1} \mathrm{em}$ bryos. (Red asterisk) HthFL; (black asterisk) HDless; (gray asterisks) additional HDless isoforms are observed in $h t h^{100-1}$ extracts, probably as a result of the premature stop codon in exon 9. (Bottom) Blue Coomassie staining of a portion of the same membrane showing equivalent loading. (B) Immunoprecipitation of total embryonic extracts with either preimmune serum (mock IP) or anti-Exd antibody (Exd IP). The blot was probed with either an $\alpha$-Hth (left panel) or an antibody raised specifically against the $\mathrm{HD}$ and C-tail of Hth $(\alpha-$ HthHD; right panel). Both full-length and HDless variants of Hth are coimmunoprecipitated with Exd, but only the larger isoform is detected with the anti-HthHD antibody. Equivalent amounts of extracts were loaded (data not shown). The additional band observed in both mock and Exd IPs in the right panel is likely due to cross-reactivity with the antibody used for the IP. $(C)$ Wing imaginal discs with mitotic clones of $h t h^{\mathrm{P} 2}$ and $h t h^{100-1}$, marked by the absence of GFP (green), stained with anti-Hth and anti-Exd as indicated. $h t h^{\mathrm{P2}}$ clones have no detectable Hth staining and no nuclear Exd, while Hth and nuclear Exd are still detected in $h t h^{100-1}$ clones. Arrows point to mutant tissue. (D) Wing or haltere imaginal discs with mitotic clones of $h t h^{\mathrm{P} 2}$ and $h t h^{100-1}$, marked by the absence of GFP (green), stained with anti-HthHD and anti-Hth $6^{\prime}$ as indicated. $h t h^{100-1}$ mutant clones are devoid of the HD-containing isoforms, but still stain for the $6^{\prime}$ HDless variant. Neither form was detected in $h t h^{\mathrm{P} 2}$ mutant tissue. Arrows point to mutant tissue.
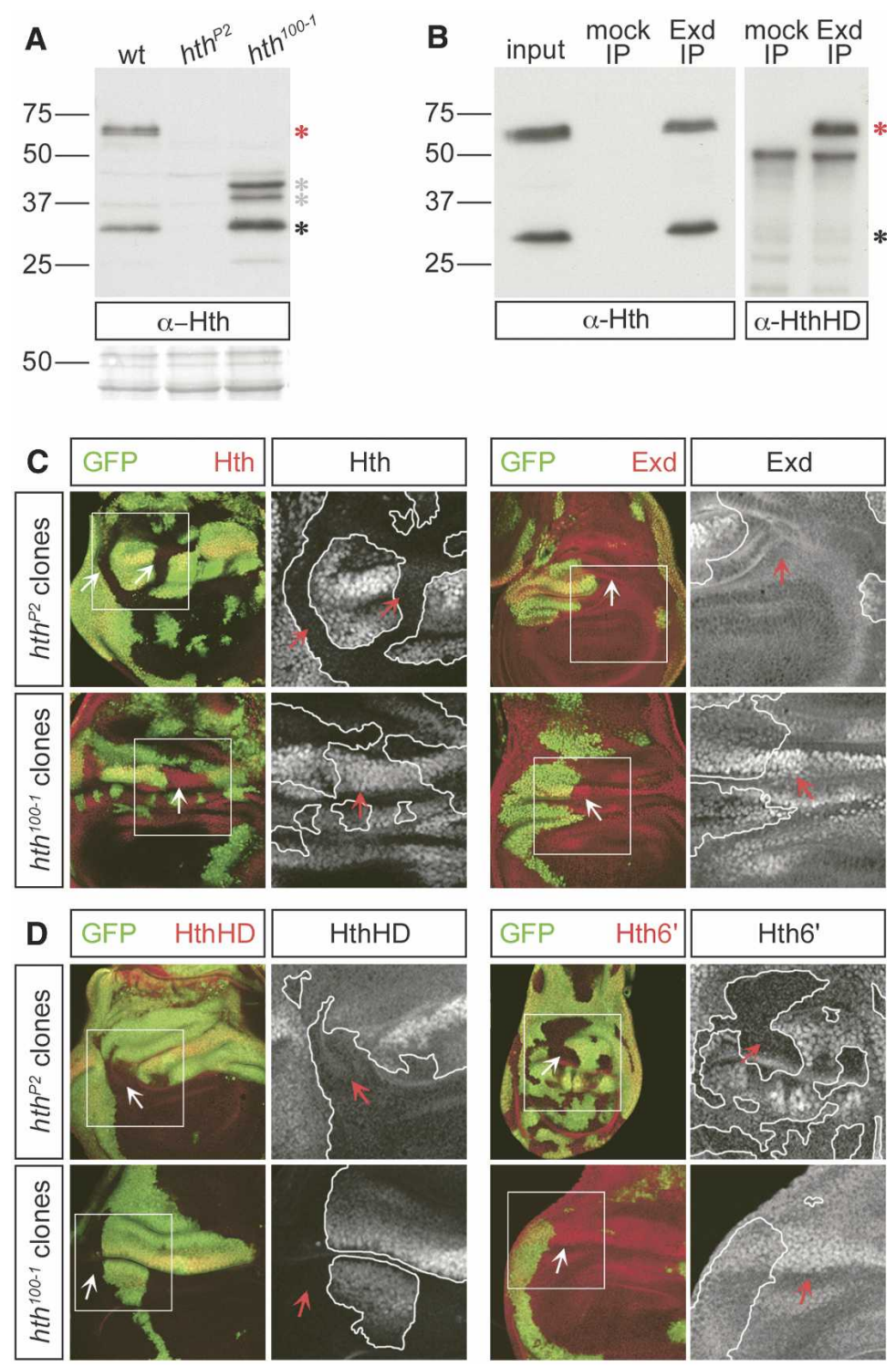

anteroposterior (AP) axis (Rieckhof et al. 1997). $h t h^{\mathrm{P} 2}$ cuticles show severe head defects, segmental fusions, and Hox-dependent, posterior-directed transformations of the abdominal segments. In the thorax, an almost completely naked epidermis with some sparse denticles replaces the rows of small denticles that are present in wild type. Transformations toward more posterior fates are evident in the first abdominal segment (A1) that takes on an A3-like identity (Fig. 3B). Denticle belt fusions, especially evident in the abdominal segments, are indicative of segmentation defects arising from the loss of engrailed (en) and wingless (wg) expression (Rieckhof et al. 1997).

When otherwise wild-type embryos were coinjected with siRNAs directed against the $6^{\prime}$ and $7^{\prime}$ isoforms, the embryos all died at the end of embryogenesis and their cuticles showed weaker versions of all of the $h t h^{\mathrm{P} 2}$ phenotypes (Supplementary Table 1). Buffer injected embryos were wild type (data not shown). In addition to head involution defects, the $6^{\prime}+7^{\prime}$ siRNA-injected embryos had a partial T1 "beard" in the second (T2) and/or third (T3) thoracic segments. This phenotype, although not observed in the complete absence of hth or exd, is seen in the absence of zygotic exd activity, a partial lossof-function situation (Peifer and Wieschaus 1990). Keilin's organs (KOs), thoracic-specific sensory structures, were often abnormal. A transformation of segmental identities was apparent in the abdomen, where A1 was partially transformed toward A2, and A2 transformed to A3, on both the ventral and dorsal surfaces (Fig. 3B; data not shown). These embryos also had segmental fusions at a frequency $(\sim 40 \%)$ comparable to $h t h^{\mathrm{P} 2}$ animals (Fig. 3B), suggesting that HDless isoforms of Hth are required for proper embryonic development. These phenotypes are likely to represent an underestimate of the role these forms play during embryonic development because the siRNA injections do not completely eliminate these mRNAs (Fig. 3A; Supplementary Fig. 2A). Moreover, 
A

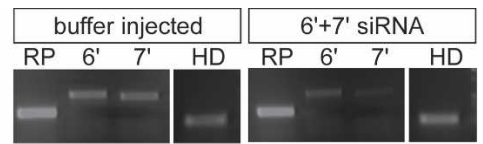

B
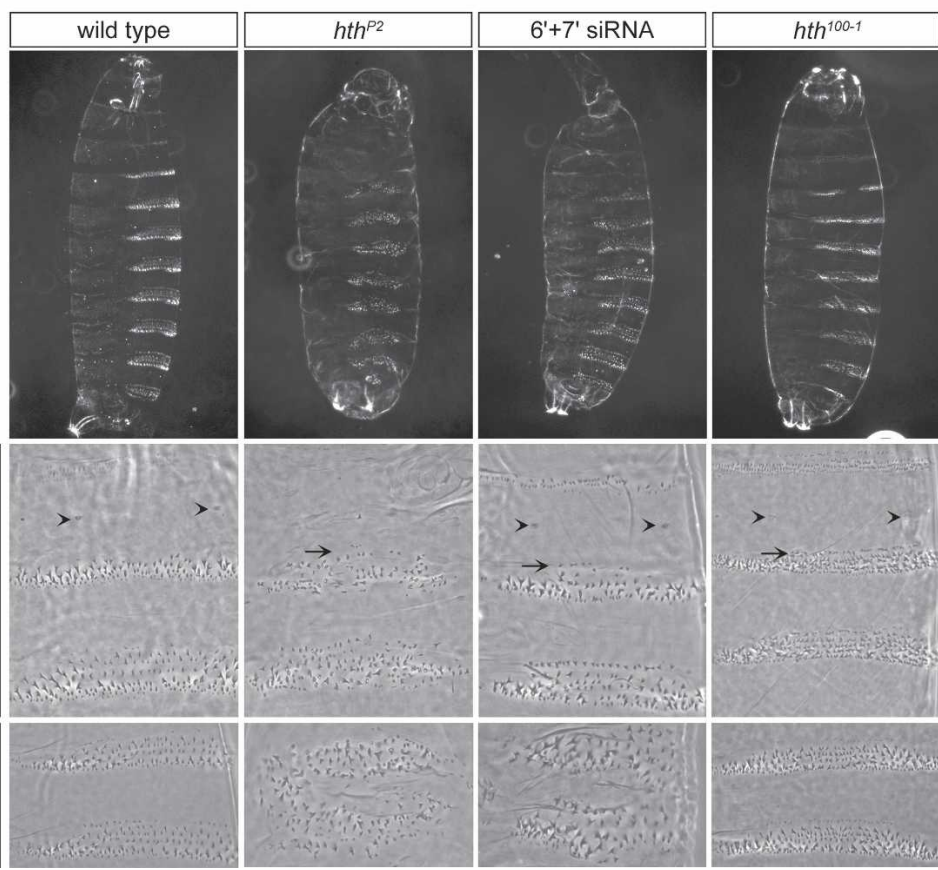

Figure 3. HDless isoforms are necessary for embryonic patterning. (A) RT-PCR from embryos injected either with buffer or siRNAs directed against the $6^{\prime}$ and $7^{\prime}$ isoforms. Specific reduction of both the $6^{\prime}$ and $7^{\prime}$ transcripts, but not the HD-containing mRNAs was observed. RT-PCR for ribosomal protein 49 transcripts $(\mathrm{RP})$ was used as a control. $(B)$ Cuticle preparations of wild-type, $h t h^{\mathrm{P} 2}, h t h^{100-1}$, and $6^{\prime}+7^{\prime}$ siRNA-injected embryos. (Top panels) Head involution defects can be observed in all mutant and siRNA-injected embryos. Strong posterior-directed transformations of the A1 and A2 segments were observed in $h t h^{\mathrm{P} 2}$ and $6^{\prime}+7^{\prime}$ siRNA-injected embryos and weaker transformations of the Al segment were observed in $h t h^{100-1}$ embryos (arrows). Arrowheads identify the KOs. (Bottom panel) Segmental fusions were observed in $h t h^{\mathrm{P} 2}$ and $6^{\prime}+7^{\prime}$ siRNA-injected embryos but not in $h t h^{100-1}$ cuticles.

these phenotypes are unlikely to be due to a general reduction in Hth levels because they are not observed in $h t h^{100-1}$ homozygous or in $h t h^{100-1} / h t h^{\mathrm{P} 2}$ embryos (see below). Indeed, a 50\% reduction in the total amount of $\mathrm{Hth}$, observable in $h t h^{P 2}$ heterozygous flies (Supplementary Fig. 2B), results in normal animals.

In contrast to the $6^{\prime}+7^{\prime}$ siRNA results, $h t h^{100-1}$ cuticle preparations are remarkably normal (Fig. 3B; Supplementary Table 1). Although head defects were apparent, the thoracic segments were normal except for the rare appearance of a small number of ectopic denticles in T3 (Fig. 3B). A1 was weakly transformed toward A2, but the rest of the abdominal segments had a wild-type pattern of denticle belts (Fig. 3B). These cuticles also lacked segmental fusions, and $e n$ and $w g$ were expressed normally (Fig. 3B; data not shown). Similar phenotypes were observed in $h t h^{100-1} / h t h^{\mathrm{P} 2}$ embryos (data not shown). These results suggest that the Hth HD is not required for proper segmentation and that it plays a relatively small role in the instruction of segmental identities. We note, however, that $h t h^{100-1}$ embryos also express HDless isoforms containing additional residues not present in the $6^{\prime}$ or $7^{\prime}$ isoforms (Fig. 2A). Although these isoforms are also HDless, they may have functions that are not present in the naturally expressed HDless isoforms. In addition, even though Hth HD is largely dispensable for some embryonic functions, HthFL isoforms are required for other Hth-dependent functions, such as peripheral nervous system development, as $h t h^{100-1}$ embryos are defective in this tissue (Kurant et al. 2001).
The Hth HD is not required for the transcriptional regulation of some direct $H$ th target genes

The dispensability of the HD for a subset of Hth functions during embryonic development suggests that distinct Hth/Exd complexes, HthFL/Exd and HDless/Exd, might control the transcriptional output of different $\mathrm{Hth}$ target genes. For some targets, Hth and Exd function as Hox cofactors, enhancing both the DNA-binding affinity and specificity of Hox proteins (Chan et al. 1994, 1996; Ryoo and Mann 1999). To assess the necessity of the Hth $\mathrm{HD}$ in the formation of functional complexes with Exd and Hox in vivo, we analyzed the expression of two previously characterized Hox/Exd target elements derived from the genes forkhead (fkh) and labial (lab), in $h t h^{100-1}$ and $h t h^{\mathrm{P} 2}$ mutant embryos.

During embryonic development, an autoregulatory enhancer from the lab gene (lab550) drives expression in a band of endodermal cells (Fig. 4A; Tremml and Bienz 1992; Grieder et al. 1997). A 48-base-pair (bp) fragment of lab550 (lab48/95) also drives expression in the endoderm and cooperatively assembles a Lab/Exd/Hth complex in vitro. Like $l a b$, both of these reporter genes require $1 a$ bial, exd, and hth for expression (Ryoo et al. 1999). For lab48/95 to be active, binding sites for all three HD proteins (Lab, Exd, and Hth) are required (Ryoo et al. 1999). In contrast, lab550, which integrates additional inputs from the decapentaplegic (dpp) pathway (Tremml and Bienz 1992; Grieder et al. 1997), does not require the Hth-binding site for activity, while it still strictly depends on both Lab- and Exd-binding sites (Ryoo et al. 

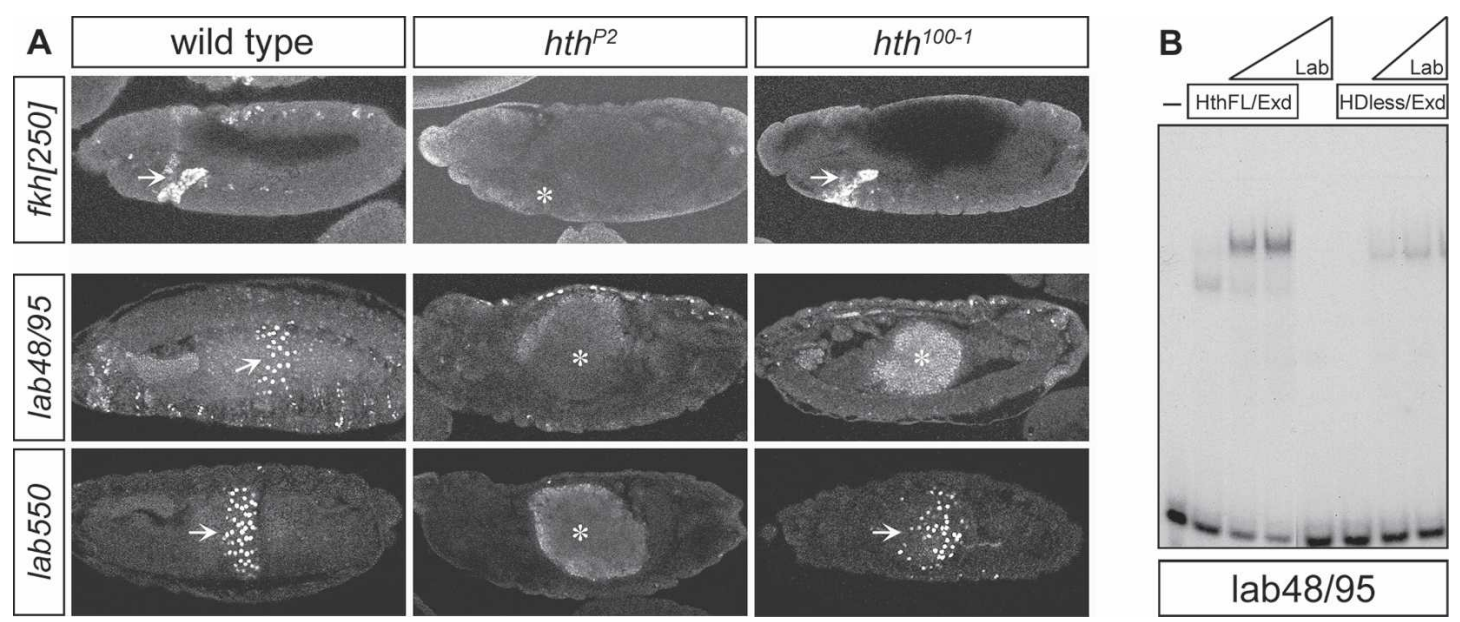

Figure 4. Differential requirement of the Hth $\mathrm{HD}$ for the transcriptional regulation of $f k h$ and $l a b .(A)$ Embryos carrying $f k h[250]-l a c Z$ (top panels), lab48/95-lacZ (middle panels), and lab550-lacZ (bottom panels), stained for $\beta$-galactosidase ( $\beta$-gal). fkh250-1acZ is expressed in wild-type and $h t h^{100-1}$ embryos, but not in $h t h^{\mathrm{P} 2}$ embryos. Wild-type embryos carrying the 1ab48/95-lacZ or 1ab550-1acZ transgenes show a very similar pattern of nuclear $\beta$-gal in a central band of endodermal cells. In hth ${ }^{100-1}$ embryos, 1ab 48/95-1acZ expression is lost, while lab550-1acZ staining is still present, underlying a differential requirement for the Hth HD by the two enhancers. In $h t h^{P 2}$ embryos, both $1 a b$ 48/95-lacZ and lab550-lacZ are lost. (Arrows) LacZ positive staining; (asterisks) no lacZ expression, although some background fluorescence is observed in these midguts. $(B)$ HDless/Exd and Lab can form a complex on the lab48/95 element in vitro, as attested by EMSA analysis. Binding is cooperative, but weaker than that observed for HthFL/Exd/Lab.

1999). These observations suggest that the Hth $\mathrm{HD}$ is not necessary for the assembly of a Lab/Exd/Hth complex in the context of the larger enhancer, probably due to the presence of additional factors involved in $1 a b 550$ activation. Consistent with these observations, lab550-1acZ is expressed normally in $h t h^{100-1}$ embryos, while lab48/95lacZ in not expressed in these embryos (Fig. 4A). These conclusions are further supported by electrophoretic mobility shift assays (EMSAs). To mimic the $6^{\prime}$ and $7^{\prime}$ isoforms found in vivo, we expressed and purified a version of Hth that contains only the HM domain, referred to here as HDless. We find that a HDless/Exd/Lab complex can cooperatively assemble on lab48/95, although approximately fourfold more weakly than HthFL/Exd/Lab (Fig. 4B). Thus, the activation of lab550 does not require a Hth-binding site or the Hth $\mathrm{HD}$, whereas the activation of $1 a b 48 / 95$ requires both.

Another Hox target element, fkh[250], derived from the gene $f k h$, is a natural target of the Hox gene Sex combs reduced $(S c r)$. Like the endogenous $f k h$ gene, fkh[250]-lacZ activation requires Scr and Exd, and these two homeoproteins bind cooperatively to this $37-\mathrm{bp}$ element in the absence of Hth (Ryoo and Mann 1999). No Hth-binding site has been found in this element, and consistently, fkh[250]-lacZ is expressed normally in $h t^{100-1}$ embryos (Fig. 4A). In contrast, fkh [250]-lacZ is no longer active in $h t h^{\mathrm{P} 2}$ embryos (Fig. 4A; Ryoo and Mann 1999). These results suggest that the HD of Hth is not required to activate the $f k h$ element but that the HM domain is necessary. Consistent with our previous observation that Exd/Scr can bind to this element in the absence of Hth (Ryoo and Mann 1999), a HDless/Exd/Scr complex can also cooperatively bind to $f \mathrm{kh}[250$ ] (data not shown). Thus, for both of these direct Hox/Exd tar- gets (lab550 and $f k h[250]$ ), the HthFL isoform is not required for activation, suggesting that the HDless isoforms are sufficient to induce the nuclear localization of Exd and activate these elements in vivo. In contrast, a weakened version of lab550 (1ab48/95) requires a Hthbinding site and the HthFL isoform for activation, suggesting that in some contexts, the Hth HD plays a role, perhaps by helping to stabilize the DNA-bound Hox/ Exd/Hth complex.

\section{The HthFL isoform is essential for antennal identity}

The experiments described above suggest, surprisingly, that the Hth HD is dispensable for many of Hth's embryonic functions. Hth and Exd are also required for many adult functions, including the specification of antennal fates and formation of the proximodistal (PD) axis of the legs and wings. Instruction of antennal identity requires the activation of antennal-specific genes, as well as the repression of leg-specific genes in the antennal imaginal disc (Casares and Mann 1998, 2001; Dong et al. 2001). To analyze post-embryonic requirements for the Hth HD, we generated $h t h^{\mathrm{P} 2}$ and $h t h^{100-1}$ clones in imaginal tissues. As previously reported, large $h t h^{\mathrm{P} 2}$ clones in the antenna result in its transformation toward a leg, containing a complete tarsus (including five segments plus the claw) and a single proximal domain (Fig. 5A; Casares and Mann 1998, 2001). The proximal domain derives from the fusion of proximal and medial leg segments, and contains both bracted and unbracted bristles, which are markers for distal and proximal leg identities, respectively. $h t h^{100-1}$ clones result in antenna-to-leg transformations that are nearly indistinguishable from those generated by $h t h^{\mathrm{P} 2}$ clones (Fig. 5A). 
A
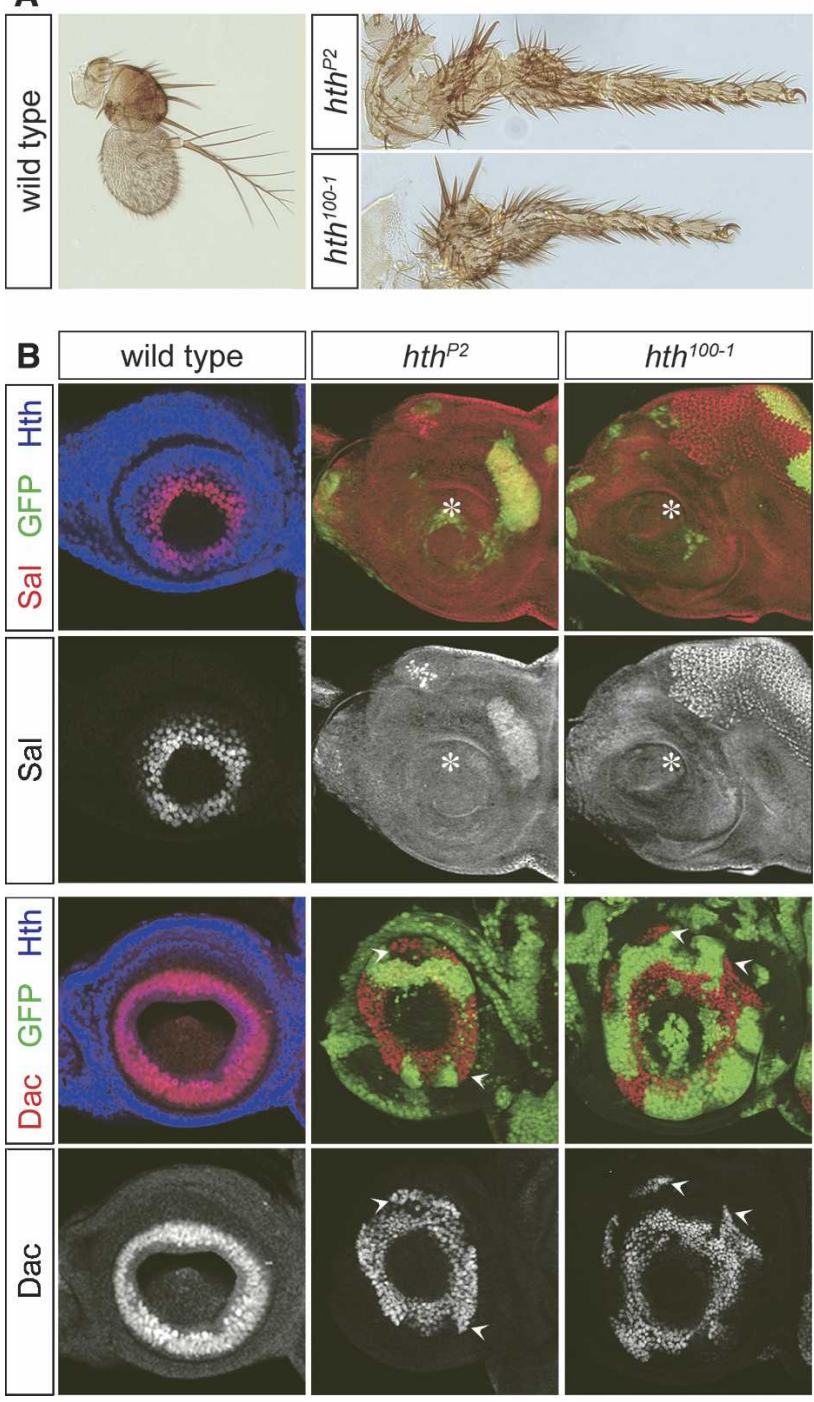

Figure 5. The Hth HD is required for the specification of antennal fates. (A) Adult wild-type, $h t h^{\mathrm{P} 2}$, and $h t h^{100-1}$ mutant antennae. Transformation of the antenna toward leg is observed in both $h t h^{\mathrm{P} 2}$ and $h t h^{100-1}$ appendages, suggesting a strict requirement for the HD in the instruction of antennal identity. (B) $h t h^{\mathrm{P} 2}$ and $h t h^{100-1}$ mitotic clones in antennal imaginal discs, marked by the absence of GFP. (Top panels) $h t h^{\mathrm{P} 2}$ and $h t h^{100-1}$ clones are devoid of Sal staining, a marker of antennal fates. Asterisks mark the mutant tissue. (Bottom panels) The Hth HD is also necessary for the repression of leg identity in the antenna, as shown by the expansion of dac expression into a leglike pattern in both $h t h^{\mathrm{P} 2}$ and $h t h^{100-1}$ mutant clones (arrowheads).

Consistent with these adult phenotypes, expression of spalt (sal), an antennal-specific marker (Dong et al. 2000), is lost in both $h t h^{\mathrm{P} 2}$ and $h t h^{100-1}$ mutant antennal imaginal discs (Fig. 5B). Conversely, ectopic expression of HthFL, but not a HDless isoform, in the antenna results in ectopic sal expression (Supplementary Fig. 3) and ectopic antennae in approximately one-fourth of the flies (see below). Thus, both loss- and gain-of-function experi- ments suggest an absolute requirement for the Hth $\mathrm{HD}$ to generate antennal fates.

To assess if the Hth $\mathrm{HD}$ is also required to repress leg identity during antennal development, we monitored the expression of dachshund (dac). dac is expressed in both leg and antenna imaginal discs but with distinct patterns (Dong et al. 2001). In the antennal disc, dac is expressed in a narrow domain, whereas in the leg, dac expression encompasses a much larger region that will give rise to medial leg segments. Both $h t h^{\mathrm{P} 2}$ and $h t h^{100-1}$ clones in the antenna result in the derepression of dac in a leg-like expression pattern (Fig. 5B). Thus, the Hth HD is not only required to activate antennal-specific genes, such as sal but is also required to repress the leg-like expression of $d a c$.

\section{The Hth HD is not required for PD axis formation or to pattern the proximal region of the ventral appendages}

Large $h t h^{\mathrm{P} 2}$ clones in the legs result in the fusion of the proximal segments, the coxa and trochanter, with medial segments and the body wall (Casares and Mann 2001; Azpiazu and Morata 2002). The resulting fused proximal domain has both bracted and unbracted bristles, suggesting it is comprised of both proximal and distal fates (Fig. 6B,D). In leg imaginal discs, $h t h^{\mathrm{P} 2}$ clones show two effects, a derepression of dac in some clones and a loss of expression of teashirt (tsh), a proximal domain marker (Fig. 6F). These phenotypes are consistent with the loss of proximal structures and the acquisition of more medial/distal identities (Abu-Shaar and Mann 1998; Wu and Cohen 2000).

In contrast to $h t h^{\mathrm{P} 2}, h t h^{100-1}$ mutant legs are normal along their entire PD axis: The coxa and trochanter are unaltered, and there are no fusions of these proximal segments with more distal leg segments (Fig. 6C,E). However, in approximately one-third of the $h t h^{100-1} \mathrm{mu}-$ tant legs, the tibia and femur were partially fused, suggesting a minor role for HthFL in separating these leg segments (data not shown). No change in tsh expression was observed and dac expression was usually unaffected in $h t h^{100-1}$ clones (Fig. 6G). Consistent with these loss-of-function phenotypes, we also found that both HthFL and HDless isoforms of Hth were able to block distal leg development when ectopically expressed in the distal domain (data not shown). These results suggest that the Hth HD is largely dispensable for PD axis formation and for specifying proximal identities in the leg.

$h$ th is also required for PD axis formation in the wing by limiting the size and position of the distal domain, the presumptive wing blade, by repressing wingless along the dorsoventral (DV) boundary (Casares and Mann 2000). As with the ventral appendages, Hth HDless isoforms are sufficient to correctly establish and maintain the PD axis in the wing disc and to limit the growth of the distal portion of the wing (Supplementary Fig. 4). In contrast to the proximal leg, however, the Hth HD is 

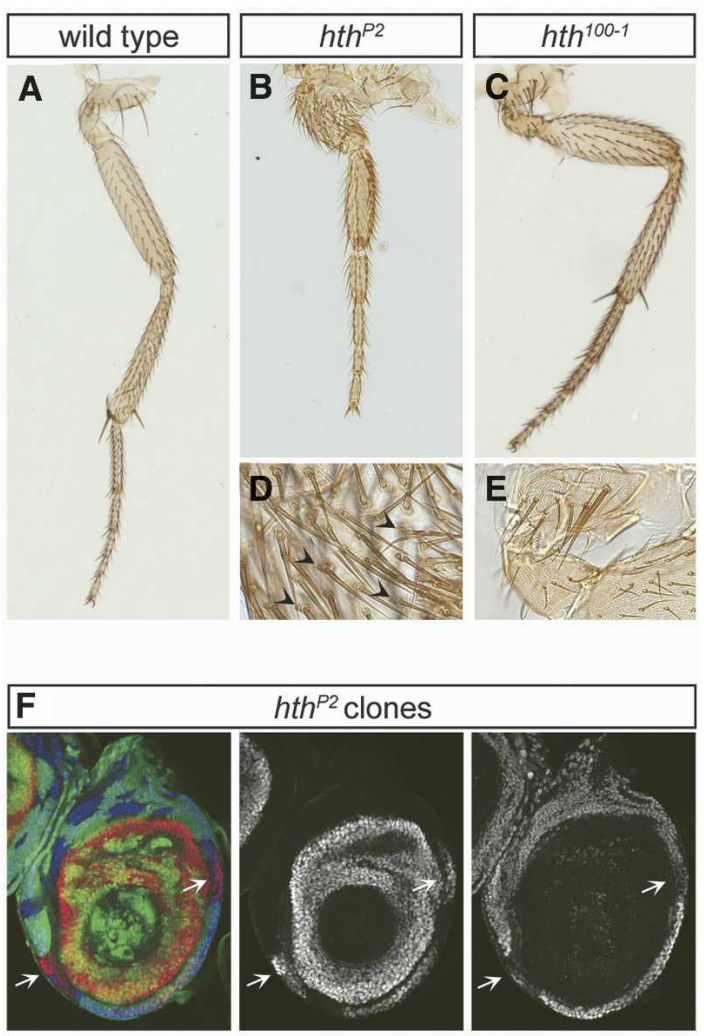

$h$ h $^{P 2}$ clones
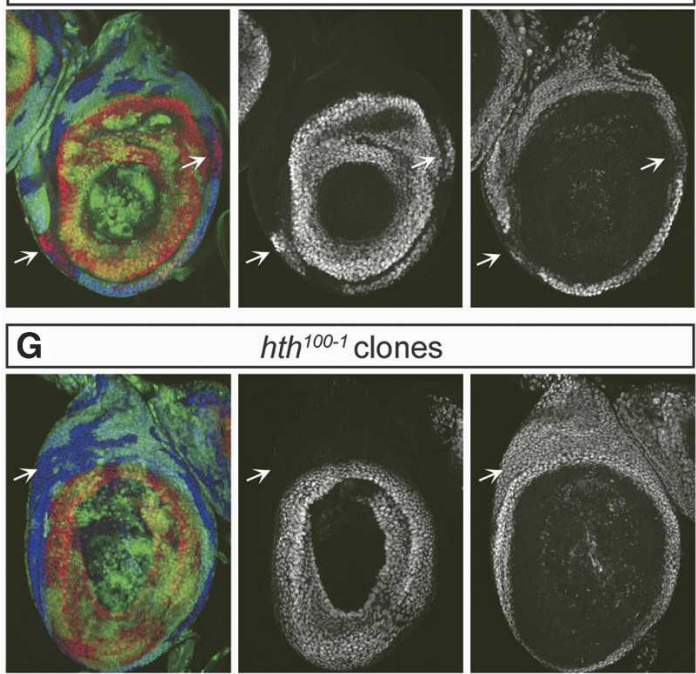

$h$ th $^{100-1}$ clones
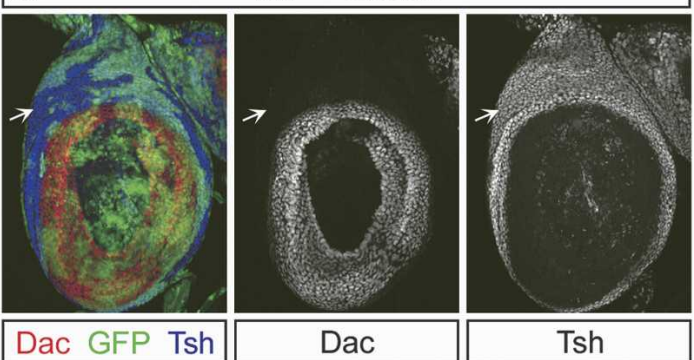

Figure 6. The Hth HD is dispensable for the formation of a correct PD axis in ventral appendages. $(A-C)$ Adult structures are shown for wild-type $(A), h t h^{\mathrm{P} 2}(B)$, and $h t h^{100-1}$ mutant legs $(C) . h t h^{\mathrm{P} 2}$ mutant legs are characterized by the loss of proximal elements, while $h t h^{100-1}$ mutant legs have a normal PD axis. $(D, E)$ High-magnification views of the proximal domains of typical $h t h^{\mathrm{P} 2}(D)$ and $h t h^{100-1}(E)$ legs. Bracted bristles, indicative of more medial/distal identities can be observed in $h t h^{\mathrm{P} 2}$, but not $h t h^{100-1}$, proximal domains (arrowheads). $(F, G)$ Mitotic clones for $h t h^{\mathrm{P} 2}(F)$ and $h t h^{100-1}(G)$ in leg imaginal discs. $h t h^{\mathrm{P} 2}$ clones show loss of $t s h$, a proximal fate marker, and the derepression of dac, a medial marker (arrows). hth ${ }^{100-1}$ clones show no loss of tsh, or derepression of dac. All clones are marked by the absence of GFP (green). Although dac expression was usually normal, we observed derepression in some $h t h^{100-1}$ clones located in the dorsal-most region of the disc (data not shown). This rare dac derepression might account for single bracted bristles in otherwise normal trochanters of some $h t^{100-1}$ legs (data not shown).

required for the proper patterning of the wing hinge, as its morphology is aberrant in $h t h^{100-1}$ clones (Supplementary Fig. 4).
Headless phenotypes generated by ectopic expression of HthFL but not a HDless isoform of Hth

The above experiments demonstrate that some Hthdependent functions, for example, antennal specification, strictly require the Hth HD while other functions, such as proximal appendage development, largely do not require HD-containing isoforms. We also find that both HthFL and HDless isoforms are coexpressed in the same cells throughout development. Taken together, these results suggest that within individual cells two sets of $h t h$ target genes are being regulated, those that depend on the $\mathrm{HD}$ for regulation and those that do not require the HD, and that these different sets of target genes have partially nonoverlapping roles in development. An additional test of this idea is to examine the consequences of overexpressing either HthFL or HDless isoforms. In principle, because there is a limiting amount of Exd, overexpression of HthFL or HDless forms should effectively reduce the amount of HDless/Exd or HthFL/Exd complexes, respectively. This prediction was confirmed by immunostaining experiments using isoform-specific antibodies in imaginal discs containing clones that ectopically express either HthFL or HDless isoforms (Fig. $7 \mathrm{~A}, \mathrm{~B})$. Expressing higher levels of one type of isoform (e.g., HthFL) lowers the levels of the other isoforms (e.g., HDless). To analyze these effects in vivo, we used a hthGal4 driver line that is only active in a subset of cells that normally express $h t h$ (data not shown). Consistent with the idea that these isoforms carry out distinct functions, hth-Gal4; UAS-HDless flies exhibit a range of phenotypes that is distinct from those observed in hth-Gal4; UAS-HthFL flies (Fig. 7C,D). In the head, the predominant phenotype resulting from HDless overexpression is an antenna-to-leg transformation $(69 \% ; n=42)$, consistent with a requirement for the HthFL isoform to make this structure. In contrast, HthFL overexpression results in a large reduction in the sizes of the eyes and head (class II + III; $\sim 93 \% ; n=63$ ). Strikingly, a significant fraction of hth-Gal4; UAS-HthFL flies have nothing left of the head except a proboscis (class III; $~ 16 \% ; n=63$ ) (Fig. $7 \mathrm{C}, \mathrm{D})$, a phenotype that was never observed in $h t h$-Gal4; UAS-HDless flies. Other phenotypes are also consistent with a division of labor between HthFL and HDless isoforms. hth-Gal4; UAS-HthFL flies often have no wing blades and no halteres $(25 \%$ and $79 \%$, respectively) whereas $h t h-G a l 4 ;$ UAS-HDless flies rarely show these phenotypes $(0 \%$ and $2.4 \%$, respectively) (Fig. $7 D$ ). Consistent with the absolute requirement for the $\mathrm{Hth} \mathrm{HD}$ in antennal development, $h t h$-Gal4; UAS-HthFL flies frequently $(24 \% ; n=38)$ have ectopic antennae in their heads, whereas hth-Gal4; UAS-HDless flies did not show this phenotype. Importantly, all of these phenotypes are significantly reverted when both HthFL and HDless forms are coexpressed using the $h$ th-Gal4 driver, which effectively restores the availability of both HthFL/Exd and HDless/Exd complexes (Fig. 7D). Thus, altering the relative levels of Hth isoforms results in distinct phenotypes, supporting our conclusion that they execute distinct functions in vivo. 


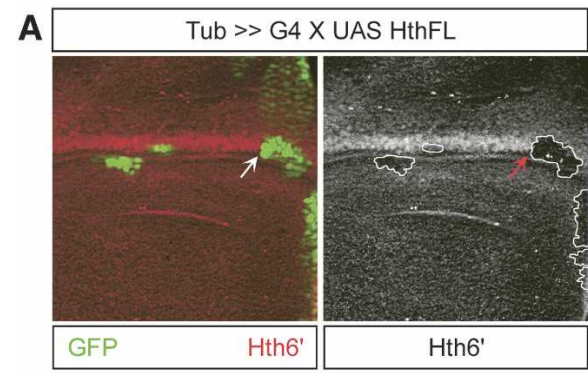

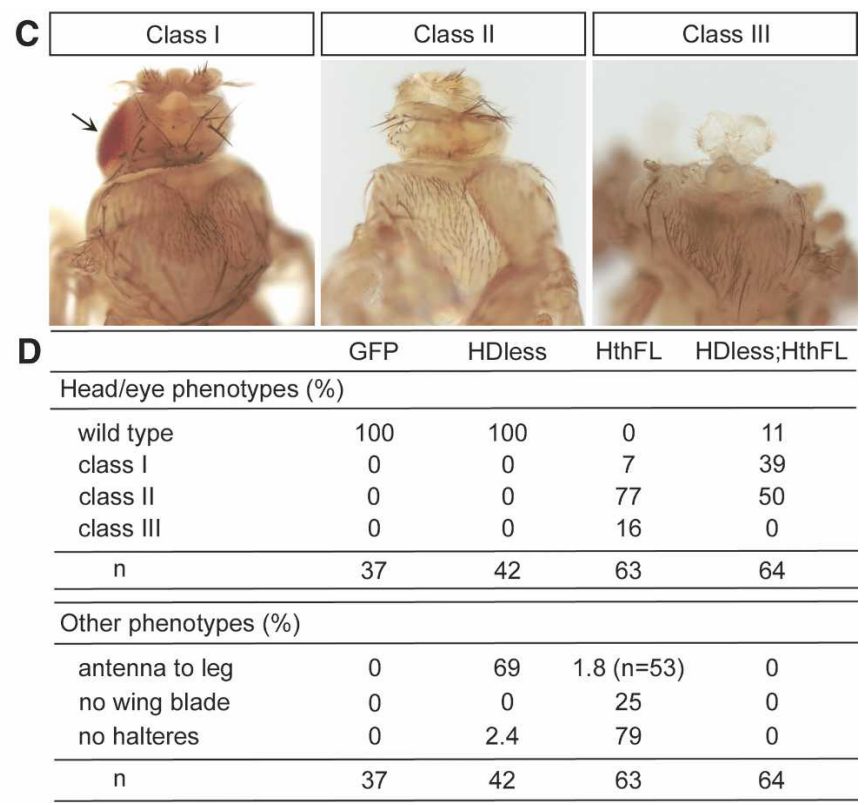

Figure 7. Altering the balance of HthFL and HDless isoforms produces unique phenotypes. $(A, B)$ Wing imaginal discs containing flip-out clones that ectopically express HthFL $(A)$ or HDless $(B)$ Hth isoforms. Clones are marked by the presence of GFP (green). Expression of HthFL leads to a reduction in the amount of endogenous HDless isoforms, as visualized by anti-Hth6' staining $(A$, arrow), while expression of HDless leads to a reduction in the amount of endogenous HthFL, as visualized by anti-HthHD staining ( $B$, arrows). $(C)$ The range of head phenotypes observed in hth-Gal4; UAS-HthFL adult flies. (Class I) Small eyes (arrow); (class II) no eyes; (class III) no head, only the proboscis remains. (D) Frequencies of phenotypes observed in $h t h-G a l 4$; UAS-GFP, $h t h-G a l 4 ;$ UAS-HDless, hth-Gal4; UAS-HthFL, hth-Gal4; UAS-HthFL, UAS-HDless adults. " $n$ " refers to the number of flies examined; for class I and II, eyes were scored individually. Because there were 10 class III (headless) flies in the HthFL experiment, only 53 flies were examined for the antenna-to-leg transformation.

\section{Discussion}

The discovery that genes are mosaics of expressed sequences (exons) embedded in a matrix of "silent DNA" (introns) raised the question of why genes are constructed in pieces (Gilbert 1978). It was quickly posited that this medley gene structure might enhance the coding capacity of the genome, as well as form an ideal framework for evolution to exploit (Gilbert 1978). Indeed, alternative splicing mechanisms have been shown to use this gene architecture to produce functionally distinct proteins from a single gene, expanding the proteome and its complexity. However, although alternative splicing can in principle lessen the discrepancy between genome coding capacity and organismal complexity, for most genes the functional differences between alternatively spliced variants have remained elusive.

In this study, we address the functional relevance of alternatively spliced isoforms of $\mathrm{Hth}$, a transcription factor involved in a wide variety of developmental programs that are critical for the construction of the D. melanogaster body plan. We report that $h$ th encodes for both HD-containing and HDless variants, both capable of forming functional $\mathrm{Hth} /$ Exd complexes with putative distinct transcriptional properties. Analysis of $h t h^{100-1}$ mutant tissues during both embryonic and larval stages have demonstrated a strict requirement for the HD in a surprisingly small subset of developmental functions, such as the instruction of antennal identity and the correct patterning of the wing hinge. In contrast, partial loss of function of HDless forms, resulting either from siRNA injection against the $6^{\prime}$ and $7^{\prime}$ isoforms or from the ectopic expression of HthFL, suggest that these forms carry out crucial functions in vivo. Intriguingly, our data further suggest that HthFL is apparently unable to substitute for at least a subset of HDless functions. This idea rests primarily on our observation that $6^{\prime}+7^{\prime}$ siRNA-injected embyros exhibit $h t h$ loss-of-function phenotypes yet still express HthFL. However, we cannot exclude the possibility that the injected siRNAs might have off-target effects, even though the specificity of the observed phenotypes suggests that it is unlikely. In future experiments, it may be possible to more definitively test this idea by generating $h t h$ mutant alleles that are unable to express the $6^{\prime}$ and $7^{\prime}$ isoforms.

On the other hand, the generally weak phenotypes observed in $h t h^{100-1}$ embryos and adults support the hypothesis that HDless/Exd dimers work as bona fide transcription factors that are essential for the correct regulation of many hth-dependent functions. In some respects, these findings are reminiscent of reports showing that an artificially truncated and HDless version of the segmentation protein Fushi tarazu (Ftz) retains many of the activities of full-length Ftz (Fitzpatrick et al. 1992; Copeland et al. 1996). These earlier findings provide addi- 
tional support to the idea that HDless forms of some homeoproteins retain biological activity, probably due to their ability to assemble stable protein complexes in vivo. What is unique to our results is that hth normally expresses HDless isoforms and that there is a division of labor between HDless and HthFL isoforms. This is best exemplified by our finding that HthFL isoforms are essential for antennal development but largely dispensable for proximal leg development. Based on these observations, we suggest that distinct Hth/Exd dimers may bind to partially overlapping sets of target genes in vivo, and that the presence or absence of the DNA-binding HD expands the range of target genes that Hth/Exd can select and regulate.

\section{Alternative splicing as a means to expand $\mathrm{Hth}$ functional diversity}

Hth is composed of two conserved modules: the HM domain that mediates an interaction with Exd and the DNA-binding HD. As the $6^{\prime}$ and $7^{\prime}$ isoforms do not have a $\mathrm{HD}$, they are unable to directly interact with DNA. However, the presence of the HM domain allows them to complex with Exd, whose HD can mediate DNA binding, as demonstrated by the formation of cooperative HDless/Exd/Hox complexes on the fkh[250] and lab48/ 95 elements. Consistent with our results, Meis has also been shown to form trimeric complexes with $\mathrm{Pbx}$ and Hox without binding directly to the DNA (Shanmugam et al. 1999).

The absence of the Hth HD has several implications for the transcriptional properties of HDless/Exd complexes. First, it is likely that HDless/Exd and HthFL/Exd complexes have distinct DNA-binding specificities because the latter complex contains two HDs, while the former contacts DNA exclusively through Exd's HD. We imagine that the two types of complexes regulate partly overlapping sets of target genes by decoding different cisregulatory architectures, possibly in the same cells. For example, the HthFL isoform appears to be unable to carry out some $h$ th functions since the $6^{\prime}+7^{\prime}$ siRNAinjected embryos exhibit hth loss-of-function phenotypes. This observation suggests that the presence of the HD might be incompatible with a subset of the cis-regulatory architectures that bind HDless/Exd. Second, HDs can also be protein interactions motifs, raising the possibility that the absence of the Hth HD from HDless/Exd could influence its ability to contact other transcription factors, coactivators, and/or corepressors. The Exd and Pbx TALE HD mediate direct interactions with Hox factors (Passner et al. 1999; Piper et al. 1999; LaRonde-LeBlanc and Wolberger 2003), and with the HD-containing transcription factor Engrailed (En) (Peltenburg and Murre 1996; Gebelein et al. 2004). The HD of Hth, which is also of the TALE family, is also likely to interact with other transcription factors, including Hox proteins (Gebelein et al. 2004). Thus, through alternative splicing, the modular architecture of $\mathrm{Hth}$ is exploited to produce unique transcription factor complexes that are likely to have distinct protein and DNA-binding properties.
The HD is required for Hth to function as a selector gene

Given that HthFL and HDless isoforms have some unique functions during development, it is tempting to suggest some generalizations about which functions require the Hth $\mathrm{HD}$ and which do not require this domain. Insect body plans are made up of repeated units that develop into diverse body parts in the adult due to the activity of selector genes, transcription factors that instruct morphological identities by regulating unique sets of target genes (Mann and Carroll 2002). Legs and antennae in Drosophila represent an example of serially homologous appendages that develop from a leg-like ground-state in response to different selector activities: Hox factors select for legs while Hth/Exd select for antenna (Struhl 1981, 1982; Casares and Mann 1998, 2001). Our demonstration that a $h t h^{100-1}$ mutant antenna is completely transformed toward a ground-state leg-like appendage demonstrates that the antennal selector function of Hth is absolutely dependent on its HD.

In contrast to its antennal selector role, our data suggest that the Hth HD is largely dispensable for at least some of the Hox-cofactor functions of Hth/Exd. This surprising conclusion is based in part on the cuticle phenotypes of $h t h^{100-1}$ and $6^{\prime}+7^{\prime}$ siRNA-injected larvae. Specifically, $h t h^{100-1}$ larvae show no or very weak transformations of segmental identity, whereas $6^{\prime}+7^{\prime}$ siRNA-injected larvae show clear posterior-directed transformations. Consistently, $h t h^{100-1}$ mutant embryos still express two directly activated Hox/Exd/Hth targets, fkh[250] and lab550. Repression of Distalless (Dll), which also requires direct Hox/Exd/Hth input /Gebelein et al. 2004), also occurs normally in $h t h^{100-1}$ mutant embryos (data not shown). Thus, from these diverse observations we conclude that the Hth HD is largely dispensable for the Hox-cofactor function of Hth/Exd. However, we note that there are exceptions to this generalization. Although activation of lab550 does not require the Hth $\mathrm{HD}$, activation of a weakened derivative of this enhancer, lab48/95, does require the Hth HD. Similarly, mutation of the Hth-binding site in the Dll repressor element, DllR, results in weak abdominal derepression (Gebelein et al. 2004). Taken together, these data suggest that the transcription factor complexes binding to the $l a b$ and Dll regulatory elements contain the Hth $\mathrm{HD}$, but that its presence is only required when the activity of these elements is compromised or weakened.

A third well-characterized function of Hth/Exd is its role in the establishment of the PD axis in both ventral (legs) and dorsal (wings and halteres) appendages. Our experiments suggest that the Hth HD is not required for PD axis formation or for specifying proximal identities in the legs. In the wing, the Hth HD is also apparently dispensable for forming a correct PD axis (in particular, repression of $w g$ at the DV boundary) but is partially required for specifying proximal (hinge) fates. Notably, both functions in which the Hth HD is largely dispensable (PD axis formation and Hox cofactor activity) appear to be evolutionarily ancient. Like Hth/Exd, Meis/Pbx are 
Hox cofactors and are also instrumental for establishing the PD axis of the vertebrate limb (Phelan et al. 1995; Berthelsen et al. 1998a,b; Jacobs et al. 1999; Mercader et al. 1999; Piper et al. 1999; Ferretti et al. 2000; LaRondeLeBlanc and Wolberger 2003). In contrast, the antennalspecifying activity of $\mathrm{Hth} / \mathrm{Exd}$, which requires the Hth $\mathrm{HD}$, is not known to have a vertebrate correlate. Thus, it is tempting to speculate that, in Drosophila, the Hth HD is more essential for executing evolutionarily recent, invertebrate-specific $\mathrm{Hth}$ functions and plays a less crucial, supplemental role in evolutionarily ancient Hth/Meis activities. Consistent with the idea that the HDless activities of $\mathrm{Hth}$ are ancient is our identification of an analogous HDless isoform made by Meis1 in Mus musculus, which underscores the functional relevance of HDless isoforms for the fulfillment of Hth/Meis-dependent functions during both invertebrate and vertebrate development. Interestingly, Prep2, another vertebrate gene related to $h$ th, also appears to encode both HDcontaining and HDless isoforms (Haller et al. 2004). Although the functions of these isoforms are not known, our results suggest that there may be a similar division of labor of HD-containing and HDless isoforms encoded by the Meis1 and Prep2 genes of vertebrates.

In summary, our results strongly support the idea that alternative splicing of $\mathrm{Hth}$ and its vertebrate orthologs is an evolutionarily conserved mechanism to expand the architectural diversity of Hth/Exd and Meis/Pbx transcriptional complexes. We propose that by excluding or including the $\mathrm{HD}$ of $\mathrm{Hth}, \mathrm{Hth} / \mathrm{Exd}$ complexes acquire distinct DNA-binding and protein interaction properties, which allow them to regulate different sets of target genes and execute unique developmental programs in vivo.

\section{Materials and methods}

\section{Genotypes and genetic manipulations}

Loss-of-function experiments. Mutant clones of hth were generated by flp-mediated recombination. Males yw; FRT82B $h t h^{P 2} / T M 6 B$ or yw; FRT82B $h t h^{100-1} / T M 6 B, y^{+}$were crossed to females yw, hs-flp; FRT82B ubi-GFP, M/TM6B to analyze mutant tissue during imaginal disc development. Mutant tissue was marked by the absence of GFP. The same males were crossed to females yw, hs-flp; FRT82B hs-CD2, y $\mathrm{y}^{+}$M/TM2 for the analysis of adult structures, with homozygous mutant tissue marked by y bristles. The progeny were heat-shocked for 15 min to $1 \mathrm{~h}$ at $37^{\circ} \mathrm{C}$, at $24-48,48-72,72-96$, and $96-120 \mathrm{~h}$ after egg laying (AEL).

fkh[250]-lacZ expression was analyzed in fkh[250]-lacZ; FRT82B $h t h^{\mathrm{P} 2}$ or FRT82B $h t h^{100-1} /$ Cyo-TM3 GFP embryos, with mutant embryos marked by the absence of GFP. To define the requirement of the Hth HD for lab expression, FRT82B $h t h^{100-1} /$ Суo-TM3 GFP flies were crossed to lab550-lacZ; FRT82B $h t h^{P 2} /$ cyo-TM6B or lab 48/95-lacZ, $h t h^{\mathrm{P} 2} / T M 2$. No lab48/95-lacZ staining was observed in any of the GFP-negative embryos, while lab550-1acZ staining was detected in half of the GFP-negative embryos analyzed. FRT82B $h t h^{\mathrm{P} 2} / T M 6 B$ flies were crossed to lab550-1acZ; FRT82B hth ${ }^{\mathrm{P} 2} /$ Cyo-TM6B or lab $48 / 95-Z, h t h^{\mathrm{P} 2} / \mathrm{TM} 2 . h t h^{\mathrm{P} 2}$ embryos were identified by the loss of anti-Hth staining.
Gain-of-function experiments. The Gal4/UAS system (Brand and Perrimon 1993) was used to ectopically express GFP and HthFL (Casares and Mann 1998) and/or HDless, previously denoted as Hth-HM (Ryoo et al. 1999), using the hth-Gal4 (GETDB-Gal4 Enhancer Trap Insertion database) driver line. Both HthFL and HDless are GFP tagged. Crosses were grown at $25^{\circ} \mathrm{C}$ for $12 \mathrm{~h}$ and then transferred at $29^{\circ} \mathrm{C}$ for the reminder of development.

yw hs-flp; tub > hs-CD2>Gal4 was used to drive expression of HthFL (Casares and Mann 1998) and/or HDless (Ryoo et al. 1999) in clones. Larvae were heat shocked for $10-15$ min at $37^{\circ} \mathrm{C}$ and then returned to $25^{\circ} \mathrm{C}$ until dissection at mid to late third instar.

The ptc-Gal4 driver line was used to misexpress GFP, HthFL, and HDless in eye-antenna imaginal discs. All the experiments were performed at $25^{\circ} \mathrm{C}$.

Whole-mount in situ hybridizations and cuticle preparations

The probe for the detection of all $h t h$ isoforms was prepared by using a full-length $h t h$ cDNA as template, while the probe specific for the HD-containing variants includes the $h t h$ cDNA sequence starting from part of exon 9 to the end of the protein. The $6^{\prime}$ and $7^{\prime}$ variant probes include the $6^{\prime}$ and $7^{\prime}$ exons, respectively, as well as part of the following exclusive $3^{\prime}$ UTRs. The probes were labeled with digoxigenin-UTP as instructed by the manufacturer (Roche), and in situ hybridizations were performed as previously described (Tautz and Pfeifle 1989). Embryonic cuticle preparations were performed as described in Wieschaus and Nusslein-Volhard (1986).

\section{RNAi}

RNAi was performed by microinjecting preblastoderm embryos as described (Kennerdell and Carthew 1998). Twenty-onenucleotide double-stranded RNA (dsRNA) (Dharmacon, A4 option) against the $6^{\prime}$ and $7^{\prime}$ isoforms was coinjected at a final concentration of 2.5 and $5 \mathrm{mg} / \mathrm{mL}$. Buffer was injected as control.

Targets sequences used were as follows: exon6' isoform, 5'-GG AGCACATGGTCCTATAT- $3^{\prime}$; exon $7^{\prime}$ isoform, $5^{\prime}$-GCAGTTC TCTATGAATATA-3'.

\section{$R T-P C R$}

Total RNA was isolated from populations of buffer or $6^{\prime}$ and $7^{\prime}$ siRNA-injected Drosophila embryos, or E10.5 mouse embryos, using standard procedures (Trizol). Synthesis of first-strand cDNA was primed by oligo (dT) and followed by RT-PCR. Oligonucleotides sequences are available upon request. The putative mouse $6^{\prime}$ homolog was sequenced revealing a bona fide $6^{\prime}$ variant.

\section{Immunohistochemistry}

Antibodies used were as follows: rabbit anti- $\beta$-galactosidase (Cappel), mouse anti-Dac (Iowa University Hybridoma bank; Mardon et al. 1994), rabbit anti-Exd (Mann and Abu-Shaar 1996), rat anti-Sal (Barrio et al. 1999), guinea pig anti-Tsh (from G. Struhl), guinea pig anti-Vg (from G. Struhl), and mouse antiWg (4D4, University Hybridoma bank; Neumann and Cohen 1997). The guinea pig (Ryoo and Mann 1999) and the rabbit anti-Hth were raised against the full-length protein. The rabbit anti-Hth6' was produced against the $\sigma^{\prime}$ exon sequence $\mathrm{N}^{\prime}$ VSTPFAGAHGPILASYNAVVHPCS- $C^{\prime}$ and used at 1:500 dilution. The guinea pig anti-HthHD was raised against a Hth fragment containing the HD and the C-terminal tail (from K363 to the end of the protein) and used at 1:500 dilution. 


\section{Immunoblot analysis and coimmunoprecipitations}

Twenty wild-type, $h t h^{\mathrm{P} 2}$, or $h t h^{100-1}$ embryos were directly lysed in SDS loading buffer and boiled before PAGE. Proteins were transferred to PVDF membranes and probed with the guinea pig anti-Hth (1:2000). As a loading control, total proteins transferred to the membrane were stained with GelCode blue (Pierce).

Immunoprecipitations were performed using equivalent amounts of 0-12 h OregonR embryonic lysate. Embryo lysates were incubated with a rabbit anti-Exd $(1: 100)$ or a preimmune serum (1:100) and protein A/G-conjugated beads overnight at $4^{\circ} \mathrm{C}$. Beads were washed three times with RIPA buffer $(50 \mathrm{mM}$ Tris at $\mathrm{pH} 7.5,150 \mathrm{mM} \mathrm{NaCl}, 20 \mathrm{mM} \mathrm{MgCl} 2,0.5 \% \mathrm{NP}-40$ ) prior to SDS-PAGE and immunoblot analysis, using a guinea pig antiHth (1:2000) or a guinea pig anti-HthHD (1:500) antibody.

\section{Protein purification and EMSA}

EMSAs were carried out as described (Gebelein et al. 2002). The HDless construct includes the first 247 amino acids and was subcloned into pET14B (Novagen). Hox, Exd and Hth proteins used were purified as His-tagged fusions using Ni-chromatography from BL21 bacteria (Gebelein et al. 2002). Protein concentrations were determined by the Bradford assay and then confirmed by SDS-PAGE and Blue Coomassie analysis (GelCode Blue, Pierce). The amounts of Hth/Exd (50 ng/lane) and HDless/ Exd dimers used were equimolar. The amounts of Lab used in Figure 4B were 80 and 160 ng. The lab48/95 probe has been previously described (Ryoo and Mann 1999; Ryoo et al. 1999).

\section{Acknowledgments}

We thank G. Struhl, R. Barrio, the Developmental Studies Hybridoma bank, and J. Dasen for reagents; R. Carthew for helpful advice on the siRNA injections; H-W. Peng for the $h t h^{100-1}$ recombinant; members of the Mann and L. Johnston laboratories for suggestions; and $\mathrm{O}$. Hobert for comments on the manuscript. This work was supported by an NIH grant (NIGMS) to R.S.M.

\section{References}

Abu-Shaar, M. and Mann, R.S. 1998. Generation of multiple antagonistic domains along the proximodistal axis during Drosophila leg development. Development 125: 3821-3830.

Abu-Shaar, M., Ryoo, H.D., and Mann, R.S. 1999. Control of the nuclear localization of Extradenticle by competing nuclear import and export signals. Genes \& Dev. 13: 935-945.

Adams, M.D., Celniker, S.E., Holt, R.A., Evans, C.A., Gocayne, J.D., Amanatides, P.G., Scherer, S.E., Li, P.W., Hoskins, R.A., Galle, R.F., et al. 2000. The genome sequence of Drosophila melanogaster. Science 287: 2185-2195.

Azpiazu, N. and Morata, G. 2000. Function and regulation of homothorax in the wing imaginal disc of Drosophila. Development 127: 2685-2693.

- 2002. Distinct functions of homothorax in leg development in Drosophila. Mech. Dev. 119: 55-67.

Barrio, R., de Celis, J.F., Bolshakov, S., and Kafatos, F.C. 1999. Identification of regulatory regions driving the expression of the Drosophila spalt complex at different developmental stages. Dev. Biol. 215: 33-47.

Bayer, C.A., Holley, B., and Fristrom, J.W. 1996. A switch in broad-complex zinc-finger isoform expression is regulated posttranscriptionally during the metamorphosis of Drosophila imaginal discs. Dev. Biol. 177: 1-14.
Berthelsen, J., Zappavigna, V., Ferretti, E., Mavilio, F., and Blasi, F. 1998a. The novel homeoprotein Prep1 modulates PbxHox protein cooperativity. EMBO J. 17: 1434-1445.

Berthelsen, J., Zappavigna, V., Mavilio, F., and Blasi, F. 1998b. Prep1, a novel functional partner of Pbx proteins. EMBO $J$. 17: 1423-1433.

Brand, A.H. and Perrimon, N. 1993. Targeted gene expression as a means of altering cell fates and generating dominant phenotypes. Development 118: 401-415.

Burglin, T.R. 1997. Analysis of TALE superclass homeobox genes (MEIS, PBC, KNOX, Iroquois, TGIF) reveals a novel domain conserved between plants and animals. Nucleic Acids Res. 25: 4173-4180.

Casares, F. and Mann, R.S. 1998. Control of antennal versus leg development in Drosophila. Nature 392: 723-726.

. 2000. A dual role for homothorax in inhibiting wing blade development and specifying proximal wing identities in Drosophila. Development 127: 1499-1508.

- 2001. The ground state of the ventral appendage in Drosophila. Science 293: 1477-1480.

Celniker, S.E. and Rubin, G.M. 2003. The Drosophila melanogaster genome. Annu. Rev. Genomics Hum. Genet. 4: 89117.

Chan, S.K., Jaffe, L., Capovilla, M., Botas, J., and Mann, R.S. 1994. The DNA binding specificity of Ultrabithorax is modulated by cooperative interactions with extradenticle, another homeoprotein. Cell 78: 603-615.

Chan, S.K., Popperl, H., Krumlauf, R., and Mann, R.S. 1996. An extradenticle-induced conformational change in a HOX protein overcomes an inhibitory function of the conserved hexapeptide motif. EMBO J. 15: 2476-2487.

Copeland, J.W., Nasiadka, A., Dietrich, B.H., and Krause, H.M. 1996. Patterning of the Drosophila embryo by a homeodomain-deleted Ftz polypeptide. Nature 379: 162-165.

Dong, P.D., Chu, J., and Panganiban, G. 2000. Coexpression of the homeobox genes Distal-less and homothorax determines Drosophila antennal identity. Development 127: 209-216.

— 2001. Proximodistal domain specification and interactions in developing Drosophila appendages. Development 128: $2365-2372$.

Dong, P.D., Dicks, J.S., and Panganiban, G. 2002. Distal-less and homothorax regulate multiple targets to pattern the Drosophila antenna. Development 129: 1967-1974.

Ebner, A., Cabernard, C., Affolter, M., and Merabet, S. 2005. Recognition of distinct target sites by a unique Labial/Extradenticle/Homothorax complex. Development 132: 15911600.

Ferretti, E., Marshall, H., Popperl, H., Maconochie, M., Krumlauf, R., and Blasi, F. 2000. Segmental expression of Hoxb2 in $\mathrm{r} 4$ requires two separate sites that integrate cooperative interactions between Prep1, Pbx and Hox proteins. Development 127: 155-166.

Fitzpatrick, V.D., Percival-Smith, A., Ingles, C.J., and Krause, H.M. 1992. Homeodomain-independent activity of the fushi tarazu polypeptide in Drosophila embryos. Nature 356: 610612.

Gebelein, B., Culi, J., Ryoo, H.D., Zhang, W., and Mann, R.S. 2002. Specificity of Distalless repression and limb primordia development by abdominal Hox proteins. Dev. Cell 3: 487498.

Gebelein, B., McKay, D.J., and Mann, R.S. 2004. Direct integration of Hox and segmentation gene inputs during Drosophila development. Nature 431: 653-659.

Gilbert, W. 1978. Why genes in pieces? Nature 271: 501.

Glazov, E.A., Pheasant, M., McGraw, E.A., Bejerano, G., and Mattick, J.S. 2005. Ultraconserved elements in insect ge- 
nomes: A highly conserved intronic sequence implicated in the control of homothorax mRNA splicing. Genome Res. 15: 800-808.

Goeke, S., Greene, E.A., Grant, P.K., Gates, M.A., Crowner, D., Aigaki, T., and Giniger, E. 2003. Alternative splicing of lola generates 19 transcription factors controlling axon guidance in Drosophila. Nat. Neurosci. 6: 917-924.

Gonzalez-Crespo, S. and Morata, G. 1995. Control of Drosophila adult pattern by extradenticle. Development 121: 21172125.

Graveley, B.R. 2001. Alternative splicing: Increasing diversity in the proteomic world. Trends Genet. 17: 100-107.

Grieder, N.C., Marty, T., Ryoo, H.D., Mann, R.S., and Affolter, M. 1997. Synergistic activation of a Drosophila enhancer by HOM/EXD and DPP signaling. EMBO J. 16: 7402-7410.

Haller, K., Rambaldi, I., Daniels, E., and Featherstone, M. 2004. Subcellular localization of multiple PREP2 isoforms is regulated by actin, tubulin, and nuclear export. J. Biol. Chem. 279: 49384-49394.

Jacobs, Y., Schnabel, C.A., and Cleary, M.L. 1999. Trimeric association of Hox and TALE homeodomain proteins mediates Hoxb2 hindbrain enhancer activity. Mol. Cell. Biol. 19: 5134 5142 .

Kennerdell, J.R. and Carthew, R.W. 1998. Use of dsRNA-mediated genetic interference to demonstrate that frizzled and frizzled 2 act in the wingless pathway. Cell 95: 1017-1026.

Knoepfler, P.S., Calvo, K.R., Chen, H., Antonarakis, S.E., and Kamps, M.P. 1997. Meis1 and pKnox1 bind DNA cooperatively with Pbxl utilizing an interaction surface disrupted in oncoprotein E2a-Pbx1. Proc. Natl. Acad. Sci. 94: 1455314558.

Kurant, E., Eytan, D., and Salzberg, A. 2001. Mutational analysis of the Drosophila homothorax gene. Genetics 157: 689-698.

LaRonde-LeBlanc, N.A. and Wolberger, C. 2003. Structure of HoxA9 and Pbx1 bound to DNA: Hox hexapeptide and DNA recognition anterior to posterior. Genes \& Dev. 17: 20602072.

Lopez, A.J. 1995. Developmental role of transcription factor isoforms generated by alternative splicing. Dev. Biol. 172: 396411.

Maconochie, M.K., Nonchev, S., Studer, M., Chan, S.K., Popperl, H., Sham, M.H., Mann, R.S., and Krumlauf, R. 1997. Cross-regulation in the mouse HoxB complex: The expression of Hoxb2 in rhombomere 4 is regulated by Hoxb1. Genes \& Dev. 11: 1885-1895.

Maniatis, T. and Tasic, B. 2002. Alternative pre-mRNA splicing and proteome expansion in metazoans. Nature 418: 236243.

Mann, R.S. and Abu-Shaar, M. 1996. Nuclear import of the homeodomain protein extradenticle in response to $\mathrm{Wg}$ and Dpp signalling. Nature 383: 630-633.

Mann, R.S. and Carroll, S.B. 2002. Molecular mechanisms of selector gene function and evolution. Curr. Opin. Genet. Dev. 12: 592-600.

Mardon, G., Solomon, N.M., and Rubin, G.M. 1994. dachshund encodes a nuclear protein required for normal eye and leg development in Drosophila. Development 120: 3473-3486.

McGinnis, W. and Krumlauf, R. 1992. Homeobox genes and axial patterning. Cell 68: 283-302.

Mercader, N., Leonardo, E., Azpiazu, N., Serrano, A., Morata, G., Martinez, C., and Torres, M. 1999. Conserved regulation of proximodistal limb axis development by Meis1/Hth. Nature 402: 425-429.

Neumann, C.J. and Cohen, S.M. 1997. Long-range action of Wingless organizes the dorsal-ventral axis of the Drosophila wing. Development 124: 871-880.
Pai, C.Y., Kuo, T.S., Jaw, T.J., Kurant, E., Chen, C.T., Bessarab, D.A., Salzberg, A., and Sun, Y.H. 1998. The Homothorax homeoprotein activates the nuclear localization of another homeoprotein, extradenticle, and suppresses eye development in Drosophila. Genes \& Dev. 12: 435-446.

Passner, J.M., Ryoo, H.D., Shen, L., Mann, R.S., and Aggarwal, A.K. 1999. Structure of a DNA-bound ultrabithorax-extradenticle homeodomain complex. Nature 397: 714-719.

Peifer, M. and Wieschaus, E. 1990. Mutations in the Drosophila gene extradenticle affect the way specific homeo domain proteins regulate segmental identity. Genes \& Dev. 4: 12091223.

Peltenburg, L.T. and Murre, C. 1996. Engrailed and Hox homeodomain proteins contain a related $\mathrm{Pbx}$ interaction motif that recognizes a common structure present in Pbx. EMBO J. 15: 3385-3393.

Phelan, M.L., Rambaldi, I., and Featherstone, M.S. 1995. Cooperative interactions between HOX and PBX proteins mediated by a conserved peptide motif. Mol. Cell. Biol. 15: 39893997.

Piper, D.E., Batchelor, A.H., Chang, C.P., Cleary, M.L., and Wolberger, C. 1999. Structure of a HoxB1-Pbxl heterodimer bound to DNA: Role of the hexapeptide and a fourth homeodomain helix in complex formation. Cell 96: 587-597.

Popperl, H., Bienz, M., Studer, M., Chan, S.K., Aparicio, S., Brenner, S., Mann, R.S., and Krumlauf, R. 1995. Segmental expression of Hoxb-1 is controlled by a highly conserved autoregulatory loop dependent upon exd/pbx. Cell 81: 10311042.

Rauskolb, C. and Wieschaus, E. 1994. Coordinate regulation of downstream genes by extradenticle and the homeotic selector proteins. EMBO J. 13: 3561-3569.

Read, D. and Manley, J.L. 1992. Alternatively spliced transcripts of the Drosophila tramtrack gene encode zinc finger proteins with distinct DNA binding specificities. EMBO J. 11: 10351044.

Rieckhof, G.E., Casares, F., Ryoo, H.D., Abu-Shaar, M., and Mann, R.S. 1997. Nuclear translocation of extradenticle requires homothorax, which encodes an extradenticle-related homeodomain protein. Cell 91: 171-183.

Ryoo, H.D. and Mann, R.S. 1999. The control of trunk Hox specificity and activity by Extradenticle. Genes \& Dev. 13: $1704-1716$.

Ryoo, H.D., Marty, T., Casares, F., Affolter, M., and Mann, R.S. 1999. Regulation of Hox target genes by a DNA bound Homothorax/Hox/Extradenticle complex. Development 126: 5137-5148.

Schmucker, D., Clemens, J.C., Shu, H., Worby, C.A., Xiao, J., Muda, M., Dixon, J.E., and Zipursky, S.L. 2000. Drosophila Dscam is an axon guidance receptor exhibiting extraordinary molecular diversity. Cell 101: 671-684.

Shanmugam, K., Green, N.C., Rambaldi, I., Saragovi, H.U., and Featherstone, M.S. 1999. PBX and MEIS as non-DNA-binding partners in trimeric complexes with HOX proteins. Mol. Cell. Biol. 19: 7577-7588.

Struhl, G. 1981. A homoeotic mutation transforming leg to antenna in Drosophila. Nature 292: 635-638.

. 1982. Genes controlling segmental specification in the Drosophila thorax. Proc. Natl. Acad. Sci. 79: 7380-7384.

Tautz, D. and Pfeifle, C. 1989. A non-radioactive in situ hybridization method for the localization of specific RNAs in Drosophila embryos reveals translational control of the segmentation gene hunchback. Chromosoma 98: 81-85.

Tremml, G. and Bienz, M. 1992. Induction of labial expression in the Drosophila endoderm: Response elements for dpp signalling and for autoregulation. Development 116: 447-456. 
Noro et al.

Wieschaus, E. and Nusslein-Volhard, C. 1986. Looking at embryos. In Drosophila: A practical approach (ed. D.B. Roberts), pp. 199-228. IRL Press Limited, Washington, DC.

Wojtowicz, W.M., Flanagan, J.J., Millard, S.S., Zipursky, S.L., and Clemens, J.C. 2004. Alternative splicing of Drosophila Dscam generates axon guidance receptors that exhibit isoform-specific homophilic binding. Cell 118: 619633.

Wu, J. and Cohen, S.M. 1999. Proximodistal axis formation in the Drosophila leg: Subdivision into proximal and distal domains by Homothorax and Distal-less. Development 126: $109-117$.

. 2000. Proximal distal axis formation in the Drosophila leg: Distinct functions of teashirt and homothorax in the proximal leg. Mech. Dev. 94: 47-56.

Zdobnov, E.M., von Mering, C., Letunic, I., Torrents, D., Suyama, M., Copley, R.R., Christophides, G.K., Thomasova, D., Holt, R.A., Subramanian, G.M., et al. 2002. Comparative genome and proteome analysis of Anopheles gambiae and Drosophila melanogaster. Science 298: 149-159. 


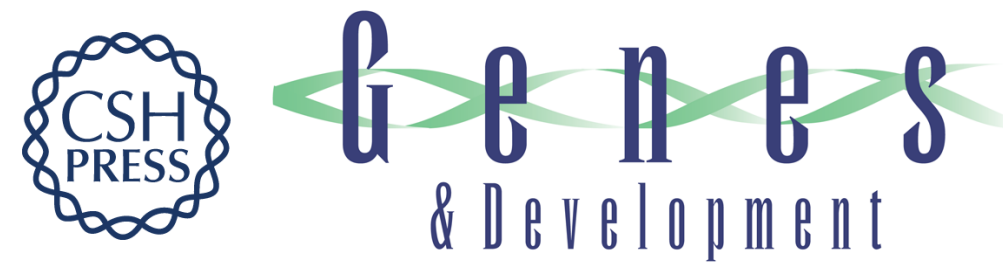

\section{Distinct functions of homeodomain-containing and homeodomain-less isoforms encoded by homothorax}

Barbara Noro, Joaquim Culi, Daniel J. McKay, et al.

Genes Dev. 2006, 20:

Access the most recent version at doi:10.1101/gad.1412606

Supplemental http://genesdev.cshlp.org/content/suppl/2006/06/01/20.12.1636.DC1
Material

References This article cites 69 articles, 33 of which can be accessed free at: http://genesdev.cshlp.org/content/20/12/1636.full.html\#ref-list-1

License

Email Alerting Receive free email alerts when new articles cite this article - sign up in the box at the top Service 